\title{
Control algorithm for multiscale flow simulations of water
}

\section{Citation}

Kotsalis, Evangelos M., Jens H. Walther, Efthimios Kaxiras, and Petros Koumoutsakos. 2009. "Control Algorithm for Multiscale Flow Simulations of Water." Physical Review E 79 (4). https:// doi.org/10.1103/physreve.79.045701.

\section{Permanent link}

http://nrs.harvard.edu/urn-3:HUL.InstRepos:41384105

\section{Terms of Use}

This article was downloaded from Harvard University's DASH repository, and is made available under the terms and conditions applicable to Other Posted Material, as set forth at http:// nrs.harvard.edu/urn-3:HUL.InstRepos:dash.current.terms-of-use\#LAA

\section{Share Your Story}

The Harvard community has made this article openly available.

Please share how this access benefits you. Submit a story.

Accessibility 


\title{
Control algorithm for multiscale flow simulations of water
}

\author{
Evangelos M. Kotsalis, ${ }^{1}$ Jens H. Walther, ${ }^{1,2}$ Efthimios Kaxiras, ${ }^{3}$ and Petros Koumoutsakos ${ }^{1}$ \\ ${ }^{1}$ Chair of Computational Science, ETH Zurich, $\mathrm{CH}-8092$, Switzerland \\ ${ }^{2}$ Department of Mechanical Engineering, Technical University of Denmark, DK-2800 Lyngby, Denmark \\ ${ }^{3}$ Department of Physics and School of Engineering and Applied Sciences, Harvard University, Cambridge, Massachusetts 02138, USA
}

(Received 3 December 2008; published 13 April 2009)

\begin{abstract}
We present a multiscale algorithm to couple atomistic water models with continuum incompressible flow simulations via a Schwarz domain decomposition approach. The coupling introduces an inhomogeneity in the description of the atomistic domain and prevents the use of periodic boundary conditions. The use of a mass conserving specular wall results in turn to spurious oscillations in the density profile of the atomistic description of water. These oscillations can be eliminated by using an external boundary force that effectively accounts for the virial component of the pressure. In this Rapid Communication, we extend a control algorithm, previously introduced for monatomic molecules, to the case of atomistic water and demonstrate the effectiveness of this approach. The proposed computational method is validated for the cases of equilibrium and Couette flow of water.
\end{abstract}

DOI: 10.1103/PhysRevE.79.045701

PACS number(s): 47.11.St

Water is the most important solvent and an ubiquitous component of biological systems. The interaction of water with its environment and the ability to capture its behavior at all scales from atomistic to continuum is challenging and has stimulated much interest in multiscale computational approaches, beginning with the work of Clementi [1].

Multiscale methods, coupling continuum models to atomistic descriptions, have been largely developed for fluids described in the atomistic regime by Lennard-Jones potentials. We may distinguish multiscale methods by the way information is exchanged between the two descriptions: by a direct flux exchange [2-5] and by a Schwarz alternating method [6-9]. A number of recent works has presented extensions to more realistic systems involving, for example, polyatomic, polar molecules such as water. Multiscale simulations of liquid water include the works of de Fabritiis et al. [3] who employed the flux exchange scheme and Praprotnik et al. [10] who presented a spatially adaptive molecular resolution procedure to transition from a coarse grained to an all-atom representation.

In this Rapid Communication, we present an application of the Schwarz alternating method $[8,9]$ to perform multiscale simulations of liquid water. The key advantage of this approach, over flux-based schemes, is that it does not require the calculation of the pressure tensor; a quantity that can be difficult to compute accurately due to limited spatial or temporal resolution. At the same time, Schwarz algorithms require several iterations in order to ensure an approximate conservation of mass and momentum between the different descriptions whereas flux-based schemes are algebraically exact. Both schemes encounter a difficulty with density oscillations attributed to the removal of periodic boundary conditions (BCs) in the atomistic domain. Flux-based schemes employ a buffer region that should be sufficiently large to ensure bulk properties in the atomistic region [3]. In Schwarz algorithms, a boundary force is introduced, along with a specular wall, to account for the missing component of the virial pressure and to eliminate these density oscillations. In this Rapid Communication, we determine the boundary force for multiscale simulations of liquid water, by extending the control algorithm, previously developed for monatomic liquids [8] to the case of water.

We begin with a summary of the general Schwarz iteration procedure (see Fig. 4). In this approach, a solution of the continuum velocity field is obtained first subject to external (outer boundary) and internal (from the atomistic region) boundary conditions. This is followed by a solution of the atomistic scale equations, usually Newton's equations of motion as embodied in molecular-dynamics (MD) methods. This is implemented in the following five steps. (a) The interactions between atoms are computed, including the boundary force $F_{m}$. (b) The velocity boundary condition obtained from the continuum solution is imposed on the atomic degrees of freedom. (c) The atomistic-continuum interfaces are moved with the local continuum velocity normal to the interface to allow flow inward and outward of the atomistic domain; the atoms that have crossed the interfaces are bounced and the interface positions are reset to the initial values to keep a constant frame of reference. (d) The particles that have left the atomistic domain are reinserted $[11,12]$. (e) A velocity boundary condition, as measured in the atomistic domain, is constructed for the continuum region and used for the next iteration. The steps are then repeated again, starting with the solution of the continuum velocity field.

In the present algorithm, the atomistic region is described by MD simulations subject to nonperiodic boundary conditions (NPBCs). The position $\mathbf{r}_{i}=\left(x_{i}, y_{i}, z_{i}\right)$ and velocity $\mathbf{u}_{i}$ $=\left(u_{i}, v_{i}, w_{i}\right)$ of the $i$ th particle evolve according to the Newton's equation of motion,

$$
\begin{gathered}
\frac{d}{d t} \mathbf{r}_{i}=\mathbf{u}_{i}(t), \\
m_{i} \frac{d}{d t} \mathbf{u}_{i}=\mathbf{F}_{i}=-\sum_{j \neq i} \nabla U\left(r_{i j}\right),
\end{gathered}
$$

where $m_{i}$ is the mass and $\mathbf{F}_{i}$ is the force on particle $i$. The interaction potential $U\left(r_{i j}\right)$ models the physics of the system. 


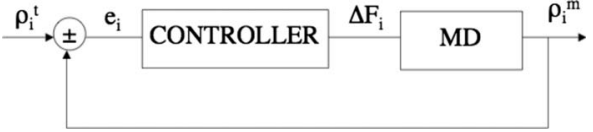

FIG. 1. Schematic representation of the control algorithm for reducing density fluctuations. The controller uses the error $e_{i}$, which is the difference between the target density $\rho_{i}^{t}$ and the computed density $\rho_{i}^{m}$, to obtain a correction to the boundary force $\Delta F_{i}$, which is applied to the MD boundary to produce the correct density and virial component of the pressure; the procedure is iterated until the error is smaller than a prescribed tolerance.

Here we consider liquid water modeled using the rigid simple point charge/extended water model by Berendsen et al. [13], with an O-H bond length of $1 \AA$ and a $\mathrm{H}-\mathrm{O}-\mathrm{H}$ angle of $109.47^{\circ}$ constrained using the SHAKE algorithm [14]. The long-range electrostatic interactions are treated using the reaction field method [15]. The nonbonded interactions are computed using a molecular cutoff $\left(r_{c}\right)$ of $1.0 \mathrm{~nm}$ and the equations are integrated using a leap-frog scheme with a time step $\delta t=2$ fs.

We impose NPBC with a boundary force $F_{m}$ to exert the correct mean virial pressure on the MD system [8]. A specular boundary is used to prevent molecules from leaving the atomistic domain and to impose the ideal kinetic part of the system pressure. The collisions are detected in a moving frame of reference. At the end of the time step, the moving boundaries are reset to their initial position and particles that end up outside the computational domain are then reinserted $[11,12]$. More specifically, we first detect the collision of a water molecule by computing whether its center of mass has crossed the boundary; thus, at each time step $n$, we compute the collision time as $t^{\prime}=\left(x_{c}^{n}-x_{b}\right) /\left(u_{b}-\widetilde{u}_{c}^{n+1 / 2}\right)$. Here, $x_{c}^{n}$ is the position of the center of mass of the water molecule, $x_{b}$ and $u_{b}$ are the initial boundary position and boundary speed, and $\tilde{u}_{c}^{n+1 / 2}$ is the center-of-mass velocity of the molecule after the regular leap-frog update but before a possible reflection. Note that we are only concerned with molecular positions $x_{i}$ and velocities $u_{i}$ in the direction perpendicular to the boundary and have dropped the molecule index $i$ for simplicity. If $t^{\prime}$ is smaller than the time step $\delta t$ then the molecule is crossing the boundary and the new velocity and position of each atom of the molecule are calculated as

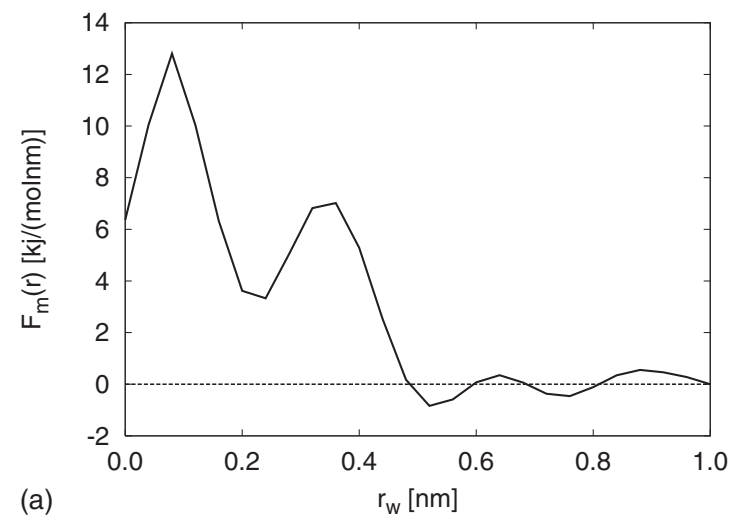

FIG. 2. (a) The external boundary force computed after applying dynamic control theory. (b) The corresponding reduced density $\left(\rho^{+}=\rho / \rho_{\text {bulk }}\right)$ values, without the control (zero external boundary force, dotted line) and with the control (solid line). For these simulations, we used $k_{p}=\mathrm{nm}^{2.5} \mathrm{~kJ} / \mathrm{amu} \mathrm{mol}$ and both the force and density were sampled over $0.36 \mathrm{~ns}$.
In [8] the boundary force $F_{m}$ is computed from the pair potential and the pair-correlation function $g(r)$ of the fluid. This approach was shown to be superior to existing approaches but is not sufficient for dense liquids at low temperatures as the pair-correlation function may not be sufficient to describe the structure of the liquid. In [9] a control algorithm was introduced in order to achieve the target density of the system for a wide range of temperatures. This algorithm is here extended to the case of water described by a fully atomistic model.

We apply our method to a model water system at equilibrium in the liquid phase, namely, $(T=300 \mathrm{~K}, \rho$ $\left.=0.997 \mathrm{~g} \mathrm{~cm}^{-3}\right)$. The size of the computational domain is $3 \times 3 \times 3 \mathrm{~nm}^{3}$. Nonperiodic boundary conditions are imposed in the $x$ direction. The system is weakly coupled to a Berendsen thermostat [16] with a time constant of 0.02 ps. In order to reduce spurious oscillations in the density, we apply the control algorithm to the mean external boundary force applied to the MD system. The control approach is sketched in Fig. 1. Each iteration involves the following steps. We start by applying a zero external boundary force. The density is sampled in 3 ps time intervals and we employ a filter to reduce the signal noise. The filter reduces the required sampling size and hence improves the convergence properties. The density $\rho^{m^{\prime}}$ is measured with a spatial resolution $(\delta x)$ of $0.025 \mathrm{~nm}$ in time intervals of $3 \mathrm{ps}$ and processed twice through a Gaussian filter to obtain $\rho^{m}$,

$$
\rho^{m}(x)=\frac{1}{\epsilon^{2}} \int\left[\int \rho^{m^{\prime}}\left(x^{\prime \prime}\right) e^{-\left(x-x^{\prime \prime}\right)^{2} / \epsilon^{2}} d x^{\prime \prime}\right] e^{-\left(x-x^{\prime}\right)^{2} / \epsilon^{2}} d x^{\prime},
$$

where $\epsilon=2 \delta x$. The cutoff used for the discrete evaluation of the convolution is $3 \delta x[9]$. We then evaluate the error in the fluid density as

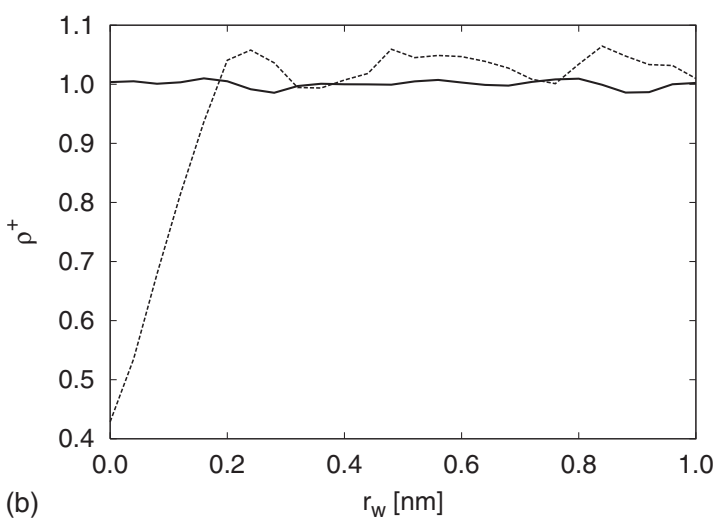

(b) 


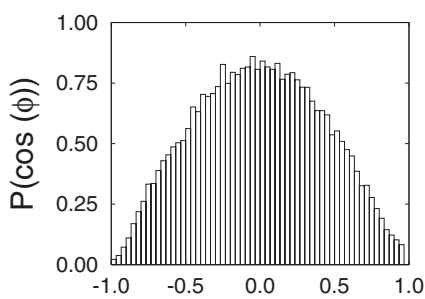

(a)

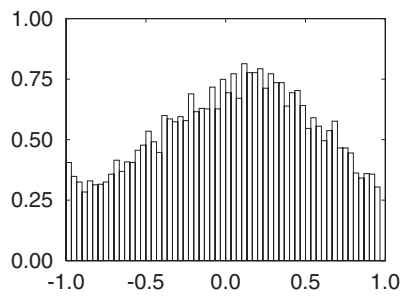

(b)
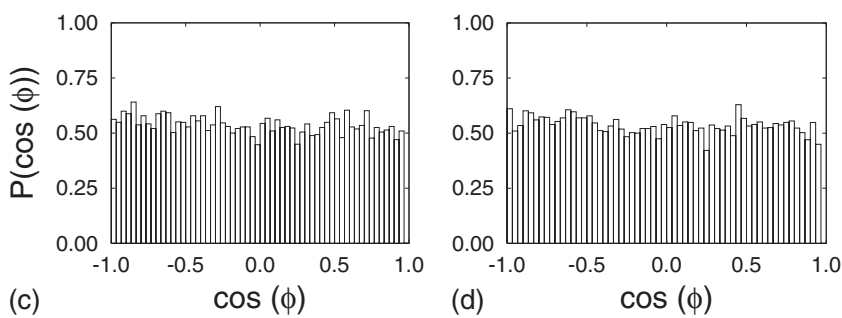

FIG. 3. The probability distribution of the cosine of the angle $\phi$ between the dipole of each water molecule with the normal to the $x$ direction at distances from the boundary: (a) $0.1 \mathrm{~nm}$, (b) $0.2 \mathrm{~nm}$, (c) $0.4 \mathrm{~nm}$, and (d) $0.6 \mathrm{~nm}$.

$$
e\left(r_{b}\right)=\rho^{t}-\rho^{m}\left(r_{b}\right),
$$

where $r_{b}$ is the distance to the boundary, $\rho^{t}$ is the desired constant target density, and $\rho^{m}$ is the measured filtered value. We compute the gradient of this error as

$$
\epsilon\left(r_{b}\right)=\nabla e\left(r_{b}\right)=-\nabla \rho^{m}\left(r_{b}\right)
$$

and amplify this with a factor $K_{i}^{P}$ to obtain the adjustment $\Delta F$ to the boundary force as

$$
\Delta F_{l}=K_{l}^{P} \epsilon_{l}
$$

for each $l$ th bin, where $K^{P}\left(r_{w}\right)=k_{p} \sqrt{\left(r_{c}-r_{b}\right)}$ and $k_{p}$ is a constant factor. We let $K^{P}$ depend on the distance from the boundary because the magnitude of the density disturbances is reduced as the distance increases. The boundary force is finally computed as

$$
F_{l}^{\text {new }}=F_{l}^{\text {old }}+\Delta F_{l},
$$

and applied to the center of mass of each water molecule (see Fig. 2). We consider that the method has converged when the root mean square of the error

$$
E=\sqrt{\frac{1}{N} \sum_{l=1}^{N} e_{l}^{2}}
$$

is less than a prescribed value, here $1 \%$. The controller is continuously acting on the system and $E$ is computed in time intervals of 90 ps.

When the external force is not acting on the system, we observe up to $60 \%$ density fluctuations. The results of applying the control shown in Fig. 2 demonstrate that our approach eliminates these density oscillations. The value of $k_{p}$ determines the stability properties and the convergence rate of the algorithm [9]. With $k_{p}=0.75 \mathrm{~nm}^{2.5} \mathrm{~kJ} / \mathrm{amu}$ mol, the convergence to $E \leq 1 \%$ is achieved after 0.4 ns. The integral [8]

$$
\rho_{n} \int_{0}^{r_{c}} F_{b}(r) d r,
$$

where $\rho_{n}$ is the number density of water, $F_{b}$ is the external boundary force, and $r$ is the distance from the boundary, which matches (to within $4 \%$ ) the correct virial pressure in the bulk. As a final check, we measure the angle $\phi$ between the normal to the boundary and the dipole moment of each water molecule. In Fig. 3 we show the angle probability distribution of $\cos (\phi)$ at different distances from the boundary. The NPBC produces a spurious preference in the orientation of the water molecules on the boundary which vanishes at a distance of approximately $0.6 \mathrm{~nm}$ from the boundary.

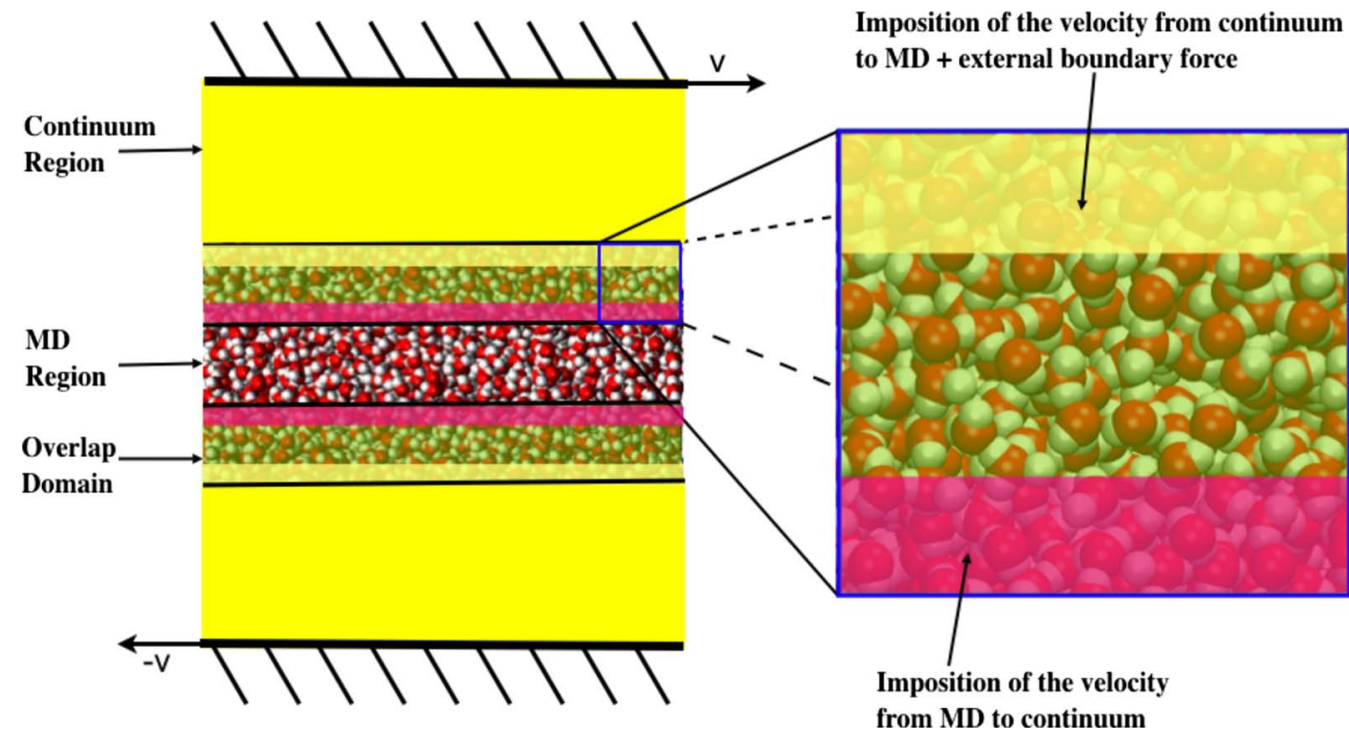

FIG. 4. (Color online) Schematic representation of the hybrid simulation for the Couette flow, indicating the atomistic region treated with $\mathrm{MD}$, the continuum region, and the motion of the boundary far from the atomistic region (with velocity $\pm v$ ). 


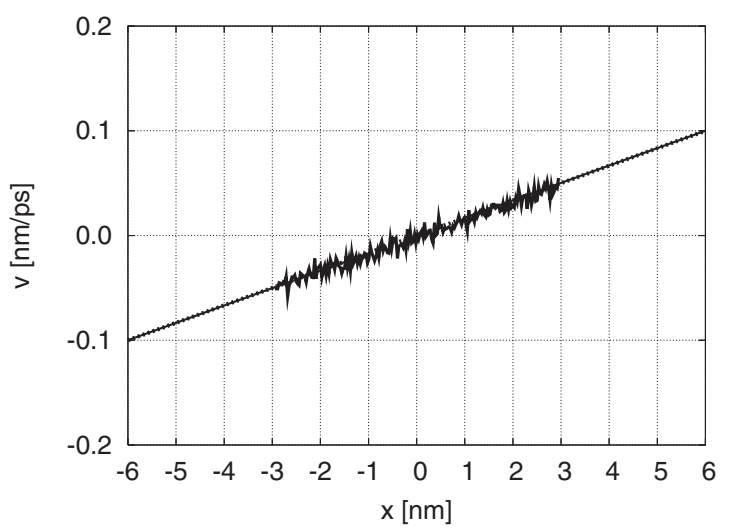

FIG. 5. Velocity profiles sampled over $1.8 \mathrm{~ns}$ in the $x$ direction in the case of Couette flow, with the hybrid method and the control algorithm applied to the atomistic region (fluctuating black line) compared to the continuum case (dashed line). In the hybrid approach, the region $-3 \mathrm{~nm} \leq x \leq 3 \mathrm{~nm}$ is treated atomistically and the regions $-6 \mathrm{~nm}<x<-1 \mathrm{~nm}$ and $1 \mathrm{~nm}<x<6 \mathrm{~nm}$ are treated by a continuum approach.

As a further test, we apply the control algorithm to the case of Couette flow. A schematic representation of the flow geometry is shown in Fig. 4. The size of the computational domain is $12 \times 3 \times 3 \mathrm{~nm}^{3}$. A resolution of $0.5 \times 3 \times 3 \mathrm{~nm}^{3}$ is used to sample the velocities that serve as a $\mathrm{BC}$ for the continuum solver. The flow is imposed by moving the upper wall with a velocity $v=0.1 \mathrm{~nm} / \mathrm{ps}$ and the lower one with $-v$. In the hybrid approach, we apply the Schwarz alternating method with an overlap region of four cells $(2 \mathrm{~nm})$ on each side, with the continuum-atomistic overlap in the regions $-3 \mathrm{~nm}<x<-1 \mathrm{~nm}$ and $1 \mathrm{~nm}<x<3 \mathrm{~nm}$. Details about the exchange of boundary conditions between the MD and the continuum region described by incompressible NavierStokes (NS) equations can be found in Ref. [8]. In the present case, the solution to the NS equations is a linear streamwise velocity profile. The MD subdomain in the hybrid case has the dimensions $6 \times 3 \times 3 \mathrm{~nm}^{3}(12$ boxes in $x$ and one box in $y$ and $z$ directions). In one cycle of the hybrid algorithm, we impose the $\mathrm{BC}$ from the continuum to the MD and subsequently sample the velocities for $80 \mathrm{ps}$ to extract the $\mathrm{BC}$ for the continuum. We sample the results for $1 \mathrm{~ns}$ and show the velocity profiles obtained from the continuum and hybrid simulations in Fig. 5; the two sets of values are in excellent agreement.

In conclusion, we have presented a control algorithm to eliminate density fluctuations in the coupling of atomistic models with continuum descriptions of liquid water. A dynamic controller based on the errors measured in the local fluid density (that may reach up to 60\%) provides an appropriate boundary forcing which applies the correct virial pressure to the system. The algorithm was applied to water at rest, in which case it eliminates the density oscillations, and to Couette flow, in which case it recovers the linear profile of the velocity field. Ongoing work aims to develop controllers that can eliminate the spurious orientation of the water molecules near the atomistic-continuum interface and the extension of the coupling to fully three-dimensional configurations.
[1] E. Clementi, Philos. Trans. R. Soc. London, Ser. A 326, 445 (1988).

[2] S. T. O'Connell and P. A. Thompson, Phys. Rev. E 52(6), R5792 (1995).

[3] G. De Fabritiis, R. Delgado-Buscalioni, and P. V. Coveney, Phys. Rev. Lett. 97, 134501 (2006).

[4] E. G. Flekkøy, G. Wagner, and J. Feder, Europhys. Lett. 52, 271 (2000).

[5] E. G. Flekkoy, R. Delgado-Buscalioni, and P. V. Coveney, Phys. Rev. E 72, 026703 (2005).

[6] N. G. Hadjiconstantinou, J. Comput. Phys. 154, 245 (1999).

[7] X. B. Nie, S. Y. Chen, W. N. E, and M. O. Robbins, J. Fluid Mech. 500, 55 (2004).

[8] T. Werder, J. H. Walther, and P. Koumoutsakos, J. Comput. Phys. 205, 373 (2005).

[9] E. M. Kotsalis, J. H. Walther, and P. Koumoutsakos, Phys.
Rev. E 76, 016709 (2007).

[10] M. Praprotnik, S. Matysiak, L. Delle Site, K. Kremer, and C. Clementi, J. Phys.: Condens. Matter 19, 292201 (2007).

[11] R. Delgado-Buscalioni and P. V. Coveney, J. Chem. Phys. 119, 978 (2003).

[12] G. De Fabritiis, R. Delgado-Buscalioni, and P. V. Coveney, J. Chem. Phys. 121, 12139 (2004).

[13] H. J. C. Berendsen, J. R. Grigera, and T. P. Straatsma, J. Phys. Chem. 91, 6269 (1987).

[14] J. P. Ryckaert, G. Cicotti, and H. J. C. Berendsen, J. Comput. Phys. 23, 327 (1977).

[15] I. G. Tironi, R. Sperb, P. E. Smith, and W. F. van Gunsteren, J. Chem. Phys. 102, 5451 (1995).

[16] H. J. C. Berendsen, J. P. M. Postma, W. F. van Gunsteren, A. DiNola, and J. R. Haak, J. Chem. Phys. 81, 3684 (1984). 